\title{
Análisis de Perfil de Textura de Ahuyama (Cucurbita maxima) sometida a Freído Atmosférico por Inmersión
}

\author{
Jhonatan A. Rodríguez-Manrique ${ }^{(1) \star}$, Armando Alvis-Bermudez ${ }^{(2)}$ y Carlos S. Cohen-Manrique ${ }^{(1)}$ \\ (1) Facultad de Ciencias Básicas, Ingeniería y Arquitectura. Corporación Universitaria del Caribe CECAR, \\ Sincelejo, Colombia. (e-mail: jhoej087@gmail.com; carlos.cohen@cecar.edu.co) \\ (2) Facultad de Ingeniería, Universidad de Córdoba, Montería, Colombia. (e-mail: aalvis2@hotmail.com)
}

${ }^{*}$ Autor a quien debe ser dirigida la correspondencia

Recibido Ene. 17, 2017; Aceptado Mar. 27, 2018; Versión final May. 21, 2018, Publicado Ago. 2018

\begin{abstract}
Resumen
Se determinó la influencia de la temperatura y tiempo de freído por inmersión sobre las características de textura de la ahuyama (un zapallo o calabaza tropical). Metodológicamente, se determinaron las condiciones proximales iniciales de la ahuyama. Luego, se realizó un proceso de freído por inmersión empleando temperaturas de $130^{\circ} \mathrm{C}, 150{ }^{\circ} \mathrm{C}$ y $170{ }^{\circ} \mathrm{C}$ y tiempos de freído de $40 \mathrm{~s}, 80 \mathrm{~s}, 120 \mathrm{~s}$ y $160 \mathrm{~s}$. Finalmente, se realizó un análisis de perfil de textura para las características de dureza, adhesividad, cohesividad, elasticidad, y masticabilidad del producto obtenido. Se concluye, que el incremento de la temperatura y el tiempo de proceso disminuyen la dureza y la adhesividad. Finalmente, para la masticabilidad las temperaturas de proceso empleadas permiten obtener productos que requerirán igual energía para ser desintegrados e ingeridos por el consumidor.
\end{abstract}

Palabras clave: textura; ahuyama; Cucurbita maximum; freído; perfil de textura

\section{Profile Analysis of Texture of Squash (Cucurbita maximum) subjected to Atmospheric Frying by Immersion}

\begin{abstract}
The influence of temperature and frying time by atmospheric immersion on the texture characteristics of the squash was determined. Methodologically, the initial proximal conditions of the squash were determined. Then, an immersion frying process was carried out using temperatures of $130^{\circ} \mathrm{C}, 150^{\circ} \mathrm{C}$ and $170^{\circ} \mathrm{C}$ and frying times of $40 \mathrm{~s}, 80 \mathrm{~s}, 120 \mathrm{~s}$ and $160 \mathrm{~s}$. Finally, a texture profile analysis was carried out for the characteristics of hardness, adhesiveness, cohesiveness, resilience and chewiness of the product obtained. It is concluded that the increase in temperature and processing time decrease hardness and adhesiveness. Finally, for chewiness, the process temperatures used made it possible to obtain products that require the same energy to be disintegrated and ingested by the consumer.
\end{abstract}

Keywords: texture; squash; Cucurbita maximum; frying; analysis of texture profile 


\section{INTRODUCCIÓN}

La ahuyama es una hortaliza que pertenece a la familia de las Cucurbitáceas, es el fruto en baya de la calabacera, se caracteriza por ser una planta herbácea y de periodicidad anual. En diversas regiones de América, Europa y Asia, se considera como una buena fuente de alimentación humana con potencialidades para la elaboración de alimentos funcionales, puesto que posee un alto valor nutricional caracterizado por aportar un alto contenido de fibra que permite mejorar el funcionamiento gastrointestinal. Además, esta hortaliza es una buena fuente de betacarotenos, ácido ascórbico y vitamina $E$, que son elementos fundamentales para la visión y el buen estado de la piel, poseen acción antioxidante y aumentan la resistencia frente enfermedades respectivamente, y por último, proporciona una gama de microelementos como el hierro, fósforo y calcio (Arturo-Suarez et al., 2016). En Colombia, la ahuyama se destina principalmente para el consumo en fresco, también se prepara cocida y es comúnmente implementada para la elaboración de cremas y sopas (Duque, 2013).

Por otro lado, aspectos como la versatilidad para el uso alimenticio y propiedades medicinales de la ahuyama, se consideran elementos potenciales que posee esta hortaliza, y que además invitan a ampliar sus posibilidades de uso en actividades de procesamiento y agroindustrialización, ello con el fin de generarle valor agregado y contribuir al desarrollo tecnológico de la misma (Rodríguez et al., 2016). En este sentido, el freído atmosférico por inmersión es una operación unitaria que puede contribuir con el desarrollo requerido por la agrocadena de la ahuyama, ya que es un procedimiento utilizado con frecuencia en establecimientos de comercialización de alimentos para consumo directo. Este proceso, se considera como uno de los métodos de cocción con mayor aceptación a nivel mundial debido a los cambios deseables que le aporta a los alimentos (Arias-Méndez et al., 2013). El freído por inmersión, se caracteriza por ser un proceso de deshidratación que utiliza cortos tiempos de cocción, esto es explicado por la rápida trasferencia de calor que se efectúa por efecto de la temperatura del aceite caliente que entra en contacto con el alimento. Además de la transferencia de calor que se efectúa en el proceso, también se despliegan fenómenos de transferencia de masa, con lo que se generan complejas reacciones químicas, de las cuales resultan atributos deseables de calidad referentes al color, sabor, olor y textura en el producto (Zhang et al., 2018). Estos cambios, se ocasionan por la aparición de una corteza crujiente que crece y se forma en la parte externa de la matriz procesada, con la cual, se regula la pérdida de humedad, la ganancia de aceite y los cambios microestructurales al interior de la matriz (Adedeji y Ngadi 2018).

Posterior al proceso de freído, uno de los atributos de calidad sensorial que usualmente evalúan los consumidores es la textura. Este parámetro puede definirse, como el conjunto de las propiedades reológicas, de estructura geométrica y de superficie de un producto, que se ve determinada por los mecanoreceptores, receptores táctiles, receptores visuales y en algunos casos receptores auditivos (Pedreschi y Moyano, 2005). Experimentalmente, se ha demostrado que los tratamientos térmicos generan cambios en la microestructura de los alimentos, y por ende modifican sus parámetros de textura. Para analizar dichos cambios y con el propósito de determinar las propiedades viscoelásticas y texturales de los alimentos, se pueden llevar a cabo pruebas de puntura y doble compresión. En este sentido, la prueba de doble compresión se conoce como "Análisis de Perfil de Textura", este ensayo se determina de forma instrumental. Actualmente, se realiza con la ayuda de un equipo analizador de textura conocido como texturómetro, se fundamenta principalmente en la doble compresión del alimento para intentar aparentar un mordisco y la acción de los dientes.

Durante este análisis, se determinan variables como la dureza, que es definida como la cantidad de fuerza maxima requerida durante la primera compresión para prensar el alimento. La dureza simula la fuerza necesaria para comprimir el alimento entre los molares o entre la lengua y el paladar imitando el primer mordisco (González et al., 2015). La cohesividad, hace referencia a fuerza de unión entre las partículas del alimento, esta característica establece el límite en que puede ser deformado el mismo hasta antes de desfragmentarse o romperse. La adhesividad, que es considerada la fuerza negativa durante el primer mordisco, seguido el primer ciclo de compresión, donde la cruceta del texturómetro sube y vuelve a su posición original, esta simboliza el trabajo requerido para separar la muestra del plato que la comprime después de la primera compresión y define la cantidad de trabajo que se requiere para despegar el alimento de alguna superficie, que por lo general, al interior de la boca es la lengua. La elasticidad, es relacionada con la altura que recupera el alimento durante el lapso de tiempo, que inicia con la finalización de la primera compresión y finaliza con el inicio del segundo ciclo de compresión. La masticabilidad, es el producto entre la dureza, la cohesividad y la elasticidad, intenta representar la cantidad de trabajo que se necesita para lograr la desintegración del alimento con la dentadura hasta ser ingerido.

Dado el panorama anterior, y con el propósito de obtener aproximaciones ideales de los parámetros texturales de los productos resultantes del proceso de fritura por inmersión, se debe considerar la selección adecuada de aspectos como el tiempo de freído, las condiciones de enfriamiento, la temperatura del medio convectivo de calentamiento, la composición, contenido de agua y cantidad de proteínas que el alimento posee, ya que 
estos parámetros están directamente relacionados con la calidad del producto final a obtener e indudablemente influyen en su posterior aceptación. Por ello, el objetivo del presente estudio fue determinar la influencia de la temperatura y el tiempo de freído por inmersión atmosférico sobre las características de los parámetros de textura de la ahuyama.

\section{METODOLOGÍA}

Para el desarrollo experimental del estudio, se adquirieron ahuyamas (Cucurbita maxima) en almacenes de cadena de la localidad de la ciudad de Sincelejo (Colombia) con características de $13 \pm 4 \mathrm{~cm}$ de diámetro y peso de $3 \pm 0,3 \mathrm{k}$ con índice de madurez comercial de $82 \pm 0,03 \%$ estimado bajo la relación de grados Brix y acidez titulable. Las ahuyamas fueron sometidas a procedimientos de recepción y acondicionamiento. Inicialmente, fueron lavadas con una solución hipoclorito de sodio en una concentración de 5000 ppm durante $1 \mathrm{~min}$. Luego, se realizaron operaciones de pelado y cortado hasta que se obtuvieron paralelepípedos de dimensiones de $1 \mathrm{~cm} \times 1 \mathrm{~cm} \times 4 \mathrm{~cm}$ con ayuda de un sacabocados. Luego, se empacaron en bolsas transparente resellables de polietileno de calibre $200 \mathrm{~mm}$, con dimensiones de $16,5 \mathrm{~cm} \times 14,9 \mathrm{~cm}$ y se introdujeron al desecador hasta iniciar los análisis posteriores.

\section{Determinación de las condiciones proximales iniciales de la ahuyama.}

Para la caracterización proximal de la ahuyama, se realizaron una serie de pruebas para medir los componentes principales como la determinación de humedad, donde se utilizó la NTC 572 hasta alcanzar peso constante, proteínas siguiendo el método estandarizado AOAC 991,20, análisis de grasa según el método AOAC 960,39, fibra cruda a través de AOAC 978,10, cenizas por el método AOAC 923,03 (AOAC 2003) y carbohidratos mediante la ecuación (1). Todos los análisis proximales, se realizaron efectuando mediciones por triplicado.

\% Carbohidratos = $100-(\%$ Humedad - \%Grasa - \%Proteínas - \%Fibra - \%Cenizas $)$

\section{Proceso de freído por inmersión.}

Para el desarrollo del proceso de freído atmosférico por inmersión, las muestras de ahuyama fueron procesadas utilizando una freidora comercial marca GSM $\AA$ de $5 \mathrm{~L}$ de capacidad y equipada con controlador de temperaturas de rango entre $5^{\circ} \mathrm{C}$ y $200^{\circ} \mathrm{C} \pm 2{ }^{\circ} \mathrm{C}$. Como medio convectivo de calentamiento, se utilizó una mezcla de aceite vegetal (soya y oleína de palma), debido a su alta estabilidad frente a la oxidación y resistencia al maltrato térmico, con antioxidante Terbutil Hidroquinona (TBHQ) y ácido cítrico como sinergista, a una relación muestra-aceite de $1: 100 \mathrm{p} / \mathrm{v}$. Las temperaturas de proceso utilizadas fueron $130{ }^{\circ} \mathrm{C}, 150{ }^{\circ} \mathrm{C}$ y $170{ }^{\circ} \mathrm{C}$, los tiempos de procesamiento empleados fueron $40 \mathrm{~s}, 80 \mathrm{~s}, 120 \mathrm{~s}$ y $160 \mathrm{~s}$. Finalizado el proceso de freído, las muestras se escurrieron en papel absorbente durante $60 \mathrm{~s}$, luego, se empacaron en bolsas resellables de polietileno transparente de calibre $200 \mathrm{~mm}$, de dimensiones de $16,5 \mathrm{~cm} \times 14,9 \mathrm{~cm}$ previamente rotuladas y se introdujeron al desecador hasta iniciar los análisis posteriores. Todas las actividades de freído se realizaron bajo presión atmosférica (aproximadamente 101,325 kPa) y por triplicado.

\section{Análisis de la pérdida de humedad y absorción de aceite.}

Para determinar la humedad final del producto después de cada tratamiento, se siguió el método oficial de la NTC 572 hasta alcanzar peso constante. La absorción del contenido de grasa se realizó según el método oficial AOAC 960.39 (AOAC, 2003). Cada uno de estos procedimientos, se realizó por triplicado. Una vez finalizados los análisis, se realizaron gráficas descriptivas de las cinéticas de absorción de aceite y pérdidas de humedad en la ahuyama sometida al proceso de freído por inmersión.

\section{Análisis de perfil de textura.}

Una vez finalizado el tratamiento de freído de las muestras, se realizó un análisis de perfil de textura (APT), siguiendo la metodología de Alvis et al., (2017), donde se utilizó un texturómetro marca TA-XT Plus Stable Micro Systems y se realizó una doble comprensión sobre las muestras con el fin de intentar simular la mordida humana, utilizando un vástago de $75 \mathrm{~mm}$ de diámetro, con $30 \%$ de deformación (estrés normal). La velocidad del cabezal utilizada fue de $1 \mathrm{~mm} / \mathrm{s}$ y un tiempo de espera de $5 \mathrm{~s}$ entre las dos compresiones. Se determinaron como variables de respuesta las características de dureza, cohesividad, adhesividad, elasticidad y masticabilidad de la ahuyama frita. Para cada tratamiento se midieron estas variables por triplicado.

Análisis estadístico de los datos.

Para el análisis de los datos, se realizó un experimento factorial bajo arreglo completamente al azar con dos 
factores, el primer factor fue la temperatura de freído con tres niveles $\left(130^{\circ} \mathrm{C}, 150^{\circ} \mathrm{C}\right.$ y $\left.170{ }^{\circ} \mathrm{C}\right)$ y el segundo factor fue el tiempo de proceso con cuatro niveles ( $40 \mathrm{~s}, 80 \mathrm{~s}, 120 \mathrm{~s}$ y $160 \mathrm{~s})$, se realizaron tres replicas para cada tratamiento para un total de 36 unidades experimentales. Además, se realizó un análisis de varianza $(p \leq 0,05)$ para detectar diferencia significativa entre los valores medios de las características del perfil de textura y un test de comparaciones múltiples de Student Newman Keuls (SNK) para obtener el mejor tratamiento conforme al comportamiento de las variables de respuesta.

\section{RESULTADOS Y DISCUSIÓN}

En la Tabla 1, se ilustran los resultados obtenidos de la caracterización proximal de la ahuyama en fresco. Dichos resultados indican que el contenido de humedad es el componente de mayor predominancia en la ahuyama, el valor obtenido es acorde con el rango entre $79 \%$ y $90 \%$ (Oloyede et al., 2012; JacoboValenzuela et al., 2011). En cuanto al contenido de carbohidratos, el valor obtenido fue superior al valor de $5,40 \%$ reportado por Márquez et al., (2002). El porcentaje de grasa tiene valores cercanos a los reportados por See et al., (2007) entre 0,07-0,16\%. El valor de proteínas es inferior al 1,33\% reportado por Quintana et al., (2018). Respecto al porcentaje de fibra, el valor resultante supera lo reportado por Jacobo et al., (2008), quienes mencionaron un intervalo de $0,56 \%-1,56 \%$ referente a fibra cruda. Lee et al., (2002) reportaron un rango de $0,57 \%-0,89 \%$ para las cenizas en esta hortaliza, la muestra estudiada está dentro de estos parámetros. Dado lo anterior, se considera la ahuyama como una materia prima potencial para la elaboración de productos fritos tipo chips.

Tabla 1: Resultados de las condiciones proximales iniciales de la ahuyama en fresco.

\begin{tabular}{|c|c|}
\hline Tipo de análisis & Valor medio \\
\hline Humedad (\%) & $87,75 \pm 0,05$ \\
\hline Carbohidratos (\%) & $7,13 \pm 0,69$ \\
\hline Proteínas (\%) & $1,13 \pm 0,06$ \\
\hline Grasa (\%) & $0,07 \pm 0,03$ \\
\hline Fibra (\%) & $3,16 \pm 0,23$ \\
\hline Cenizas (\%) & $0,76 \pm 0,30$ \\
\hline
\end{tabular}

En la Figura 1a, se ilustran los resultados obtenidos para la absorción de aceite de acuerdo a las temperaturas evaluadas, se evidencia que con el incremento del tiempo de freído, se presenta una relación directa con respecto al ingreso de aceite en la matriz procesada. Este resultado ha de esperarse, debido a que en los procesos de freído el tiempo es un factor principal que interviene en la cantidad de agua que sale y en la formación de conductos en la matriz en proceso, que posteriormente estarán disponibles para ingreso aceite (Montes et al., 2016). Por su parte, efecto contrario ocurre con las temperaturas utilizadas en el proceso, donde se evidencia notoriamente, que incrementar este factor permite obtener productos con menor contenido de aceite, es por ello, que con la temperatura de $170^{\circ} \mathrm{C}$ se obtuvieron productos con un contenido de aceite inferior.

a. Grafica de ganancia de aceite

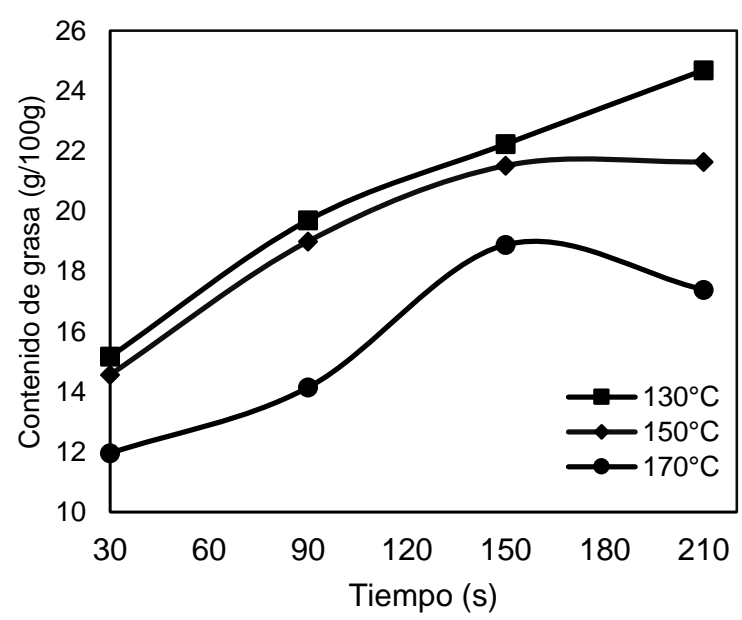

b. Grafica de pérdida de humedad

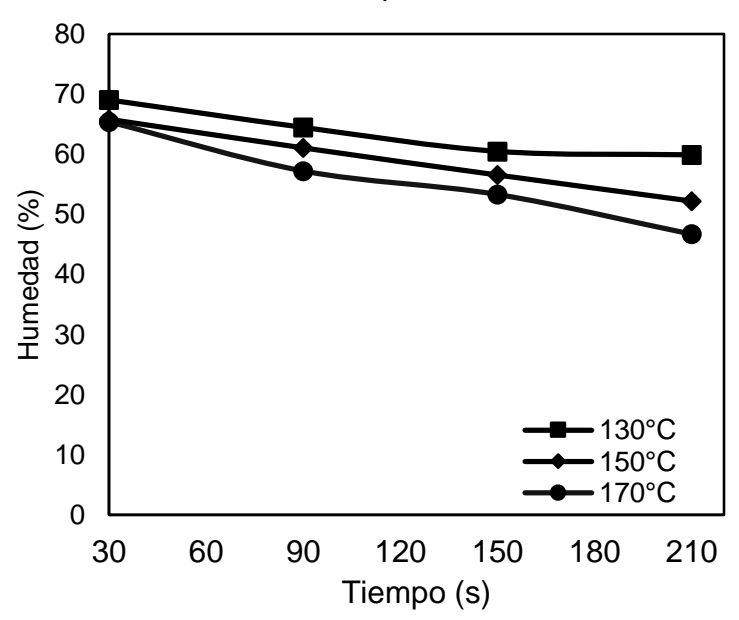

Fig. 1: Gráficos de ganancia de aceite y pérdida de humedad del proceso de freído por inmersión de la ahuyama. 
Este resultado se atribuye a que con el incremento de la temperatura, se genera mayor turbulencia por efecto de la salida del agua en forma de vapor en la superficie externa del producto, lo cual representa menor disponibilidad de aceite en la superficie externa del producto para ingresar al interior de la matriz en la etapa de final del freído, que es donde se produce una presión negativa hacia el centro del alimento permitiendo el ingreso del aceite por capilaridad (Brannan et al., 2014).

Con respecto a la pérdida de agua, el incremento de la temperatura y del tiempo de freído permiten obtener producto con menor contenido de humedad, debido a que el incremento de la temperatura genera una fuerza impulsora con capacidad superior para extraer agua desde el producto en forma de vapor (Moreno y Bouchon 2008). Debe resaltarse, en el proceso desarrollado, el incremento del tiempo en el proceso permitió la creación de un mayor número de sitios de escape del vapor en la estructura de uniones celulares en la ahuyama, con lo cual se generó un efecto positivo a favor de la pérdida de agua en la matriz procesada (Montes et al., 2016). De forma general, es posible deducir que la temperatura y el tiempo de proceso tienen una relación directa con la pérdida de agua de la ahuyama frita. Resultados similares fueron reportados por Barrios-Barrios et al., (2016) y Alvis-Bermúdez et al., (2016) en arveja y tajadas de plátano respectivamente, los estudios indicaron que el tiempo favorece la absorción de aceite en el freído por inmersión de estos alimentos. Además, con la implementación de temperaturas bajas obtuvieron el mayor contenido de aceite en los productos. Con respecto a la pérdida de humedad, el incremento de la temperatura y de tiempo de freído, disminuyen con mayor severidad esta variable en el producto final.

En la Tabla 2, se evidencia que para las variables de respuesta dureza y adhesividad la interacción doble de temperatura y tiempo indicó diferencia significativa entre los valores medios obtenidos en el análisis de perfil de textura realizado a la ahuyama frita. Ello, se atribuye a que los factores del estudio actuado de forma conjunta generan un efecto marcado sobre la estructura física del producto frito, dicho resultado es producto del efecto de la salida de agua y de la absorción de aceite en el proceso. Por su parte, para las variables de cohesividad, elasticidad y masticabilidad, las interacción doble no resulto significativa, por tanto, los factores actuando conjuntamente no ejercieron un efecto significativo que permitiera obtener diferencias con respecto a la unión de las partículas, la proporción de recuperación posterior a la primera compresión y sobre el trabajo requerido para desintegrar la ahuyama sometida al proceso de freído respectivamente. Por lo tanto, con respecto a los valores de cohesividad, se infiere que el efecto de la temperatura sobre el límite de deformación a la ruptura de la ahuyama es igual en todos los niveles del factor tiempo. De igual forma, el efecto de la temperatura no modifica significativamente la capacidad de recuperación de la ahuyama, porque no altera su estructura elástica los suficiente para marcar diferencias en sus valores medios dentro de los niveles de tiempo implementados, por tanto, la combinación de los efectos ejercidos esta variable de la ahuyama no difiere de la suma de los efectos independientes (Pardo et al., 2007). Finalmente, la combinación de los niveles de temperatura utilizados dentro de los niveles de tiempo empleados en el proceso de freído de la ahuyama no representan modificaciones marcadas en el trabajo requerido para desintegrar la ahuyama hasta deglutirla, indicando que los niveles de temperatura implementados sobre la masticabilidad de la ahuyama generó el mismo efecto en todos los niveles del factor tiempo utilizados (Barati y Talebi, 2018).

Tabla 2: Valores $p$ del análisis de varianza para las características de textura de la ahuyama frita.

\begin{tabular}{|c|c|c|c|}
\hline \multirow{2}{*}{$\begin{array}{c}\text { Variable de respuesta del } \\
\text { análisis de perfil de textura }\end{array}$} & \multicolumn{3}{|c|}{ p-valores factores e interacciones } \\
\cline { 2 - 4 } & Temperatura $\left({ }^{\circ} \mathrm{C}\right)$ & Tiempo $(\mathrm{s})$ & 0,04 \\
\hline Dureza $(\mathrm{g})$ & 0,00 & 0,00 & 0,44 \\
\hline Cohesividad & 0,00 & 0,07 & 0,02 \\
\hline Adhesividad $(\mathrm{g} \cdot \mathrm{s})$ & 0,00 & 0,90 & 0,38 \\
\hline Elasticidad & 0,85 & 0,10 & 0,51 \\
\hline Masticabilidad $(\mathrm{g})$ & 0,06 & 0,03 & Temperatura-Tiempo \\
\hline
\end{tabular}

En la Tabla 3, se muestran los resultados del test de comparaciones múltiples de Student Newman Keuls para determinar los tratamientos que presentaron diferencias en sus valores medios, considerando demarcar diferencias entre las comparaciones de los niveles de temperatura dentro de los niveles de tiempo con letras mayúsculas diferentes y demarcando diferencias entre los niveles de tiempo dentro de los niveles de temperatura con letras minúsculas. Los resultados obtenidos para la dureza indican que, con el mayor nivel de temperatura, se obtienen los valores más bajos de esta variable en todos los niveles de tiempo, revelando que la fuerza impulsora ejercida por este nivel de temperatura y el efecto de la salida de agua en forma de vapor, genera dos efectos favorables para la disminución de esta variable. De igual forma, analizando los niveles de tiempo dentro de los niveles de temperatura, se puede deducir que, con la temperatura de $130^{\circ} \mathrm{C}$ el nivel de tiempo que permitió disminuir los valores de dureza fue el de $120 \mathrm{~s}$, pero no siendo diferente estadísticamente con el nivel de $160 \mathrm{~s}$. Similar resultado, se obtiene con el nivel de temperatura de $150{ }^{\circ} \mathrm{C}$, 
donde a mayor tiempo de proceso, la dureza resultó inferior. Por último, con el nivel de $170{ }^{\circ} \mathrm{C} \mathrm{y} 160 \mathrm{~s}$ se obtuvo la menor dureza en la ahuyama, pero siendo estadísticamente igual con los otros niveles de tiempo de proceso.

Tabla 3: Resultados del Test de Student Newman Keuls (SNK) de los parámetros de textura de la ahuyama frita para los que la interacción Temperatura-Tiempo resultó significativa.

\begin{tabular}{|c|c|c|c|c|c|}
\hline \multirow{2}{*}{ Variable } & \multirow{2}{*}{\begin{tabular}{|c|} 
Factores \\
$\begin{array}{c}\text { Temperatura } \\
\left({ }^{\circ} \mathrm{C}\right)\end{array}$
\end{tabular}} & \multicolumn{4}{|c|}{ Tiempo (s) } \\
\hline & & 40 & 80 & 120 & 160 \\
\hline \multirow{3}{*}{$\begin{array}{c}\text { Dureza } \\
\text { (g) }\end{array}$} & 130 & $6918,75 \pm 1250,01^{\mathrm{a}, \mathrm{A}}$ & $6219,03 \pm 1463,90^{a}, A B$ & $4013,85 \pm 997,57^{\mathrm{ab}, \mathrm{B}}$ & $4569,24 \pm 994,20^{a, B}$ \\
\hline & 150 & $8021,37 \pm 1329,70^{a}, A$ & $4329,82 \pm 380,50^{\mathrm{ab}, \mathrm{B}}$ & $5007,80 \pm 1359,50^{a, ~ B ~}$ & $2847,54 \pm 1148,01^{a b, ~ B ~}$ \\
\hline & 170 & $3777,29 \pm 970,50^{b, A}$ & $3302,04 \pm 713,20^{\mathrm{b}, \mathrm{A}}$ & $2416,40 \pm 482,20^{\mathrm{b}, \mathrm{A}}$ & $1640,07 \pm 835,40^{\mathrm{b}, \mathrm{A}}$ \\
\hline \multirow{3}{*}{$\begin{array}{c}\text { Adhesividad } \\
(\mathrm{g} \cdot \mathrm{s})\end{array}$} & 130 & $-42,10 \pm 21,34^{a, A}$ & $-58,35 \pm 12,47^{b, A}$ & $-35,37 \pm 8,18^{a b, A}$ & $-48,65 \pm 1,83^{b, A}$ \\
\hline & 150 & $-51,49 \pm 3,85^{a, A B}$ & $-37,41 \pm 5,68^{a, A}$ & $-54,56 \pm 13,44^{b, B}$ & $-40,62 \pm 12,70^{a b,}$ AB \\
\hline & 170 & $-27,13 \pm 10,15^{a, A}$ & $-35,53 \pm 19,66^{a b, ~ A ~}$ & $-29,43 \pm 10,40^{a, A}$ & $-20,87 \pm 6,40^{\mathrm{a}, \mathrm{A}}$ \\
\hline
\end{tabular}

De forma general, puede inferirse que en el proceso de freído por inmersión de la ahuyama, la variable dureza disminuye a medida que incrementa la temperatura y el tiempo de proceso, permitiendo obtener productos con menor dureza por efecto del mayor ablandamiento por cambios en los enlaces de amilosa y amilopectina de los almidones, ocasionando mayor gelatinización cuando entran en contacto con un aceite más caliente y por mayor tiempo de exposición al intercambio másico (Granados et al., 2014). Además, con el incremento de los niveles de los factores evaluados, se genera mayor salida de agua del interior de la matriz procesada, lo cual permite obtener conductos más pronunciados por efecto de la salida del vapor, lo que indudablemente debilita la estructura del paralelepípedo al momento de la compresión y contribuye a la disminución de la dureza de la ahuyama procesada. Resultados similares congruentes con la dureza, fueron obtenidos en chips de yuca freídos atmosféricamente por Oyedeji et al., (2017), donde se observó una disminución rápida en la fuerza de ruptura máxima de la matriz alimentaria con el incremento de los parámetros del proceso, en especial con el incremento del tiempo. Dichos cambios, fueron atribuidos a cambios estructurales como la gelatinización de almidones, a la formación de la corteza crujiente y a la degradación estructura causada por la migración del agua debido al aumento de las temperaturas del aceite de freído.

Con respecto a la variable adhesividad, con el nivel de temperatura de $130^{\circ} \mathrm{C}$ y $170^{\circ} \mathrm{C}$ se obtienen valores estadísticamente iguales para representar el trabajo requerido para despegar la ahuyama frita de alguna superficie después de la primera compresión, lo cual significa que el componente adhesivo que proporciona a la matriz la captación de aceite con esta temperatura no genera diferencias significativas en los nivel tiempo evaluados, debido a que estos son muy pequeños para representar diferencias notorias. Por su parte, con el nivel de temperatura de $170{ }^{\circ} \mathrm{C}$ interactuando conjuntamente con los niveles de tiempo $40 \mathrm{~s}$ y $80 \mathrm{~s}$ y $160 \mathrm{~s}$, se obtienen ahuyamas con igual componente adhesivo. Solo se marcaron diferencias con el nivel de $120 \mathrm{~s}$ con respecto al nivel de $80 \mathrm{~s}$, pero igualdad con los demás niveles de tiempo evaluados, lo cual indica que con el nivel de tiempo de $160 \mathrm{~s}$, puede obtenerse un producto con igual componente adhesivo que con los primero niveles de tiempo, puesto que se ve influenciado por la relación obtenida entre la cantidad de aceite en la superficie del producto y la cantidad de agua que emigró hacia el exterior del alimento, permitiendo obtener un producto más seco y con adhesividad igual. De forma general, en el proceso de freído de la ahuyama bajo las condiciones experimentadas dadas, es posible inferir que el tratamiento de $170{ }^{\circ} \mathrm{C}$ de temperatura de freído interactuando con el nivel de tiempo de $160 \mathrm{~s}$, permite obtener el valor más elevado de la adhesividad, indicando productos mucho menos pegajosos que requerirán menor trabajo por parte del consumidor para lograr despegar la ahuyama frita de una superficie, ya sea de los molares o el paladar. Esto puede atribuirse a que con el incremento de los niveles de temperatura y de tiempo en el proceso, se obtienen productos más secos por efecto de la difusividad del agua en forma de vapor en la superficie externa del producto, y con ello, menos pegajosidad por efecto de la formación de la corteza crujiente resultante de la deshidratación de las células de la superficie externa de la ahuyama procesada, lo cual resulta deseable en los productos fritos (Mba et al., 2017; Kan et al., 2016).

Una vez analizada las interacciones dobles, se procedió a analizar la influencia de los factores por separado para las variables donde dicha interacción no resultó ser significativa, los resultados que se obtuvieron para este análisis se ilustran en la Tabla 4, salvo los resultados obtenidos para la elasticidad, debido a que se obtuvo que los diferentes niveles utilizados de temperatura y de tiempo actuado de forma independiente o de 
forma conjunta, no afectaron representativamente esta variable como para obtener diferencias entre ellos. Por tanto, cualquiera de las temperaturas de freído o los tiempo de proceso evaluados en este estudio, representa igual capacidad de recuperación de la altura del paralelepípedo de ahuyama frita por inmersión posterior a la primera compresión y por ello, se fragmenta igual valor de la estructura original del alimento. Este resultado se atribuye que el fenómeno de transferencia de masa efectuado sobre la ahuyama durante el proceso de freído por inmersión, no posee una relación diferenciada sobre las propiedades de estructura elástica de la ahuyama.

Tabla 4: Resultados del Test de Student Newman Keuls (SNK) de los parámetros de textura de la ahuyama frita para los que la interacción Temperatura-Tiempo no resultó significativa.

\begin{tabular}{|c|c|c|c|}
\hline \multicolumn{2}{|c|}{ Temperatura $\left({ }^{\circ} \mathrm{C}\right)$} & \multicolumn{2}{c|}{ Tiempo (s) } \\
\hline Evaluadas & Cohesividad & Evaluadas & Masticabilidad $(\mathrm{g})$ \\
\hline 130 & $0,24 \pm 0,02 \mathrm{~b}$ & 40 & $840,28 \pm 312,80 \mathrm{a}$ \\
\hline 150 & $0,32 \pm 0,06 \mathrm{a}$ & 80 & $783,57 \pm 219,80 \mathrm{ab}$ \\
\hline 170 & $0,38 \pm 0,09 \mathrm{a}$ & 120 & $744,22 \pm 333,70 \mathrm{ab}$ \\
\hline- & -- & 160 & $487,66 \pm 169,54 \mathrm{~b}$ \\
\hline
\end{tabular}

Referente a la cohesividad de la ahuyama frita, es posible observar que con las temperaturas de $150{ }^{\circ} \mathrm{C}$ y $170{ }^{\circ} \mathrm{C}$ fue donde se obtuvo el mayor valor de esta variable, revelando que después de efectuado dicho tratamiento, la ahuyama conservó la mayor unión entre las partículas que conforman la matriz, presentando la mayor resistencia a la fragmentación del producto por efecto de la acción mecánica del equipo de textura, este fenómeno se explica debido a que con la aplicación de temperaturas elevadas de procesamiento se generan modificaciones en la estructura de la ahuyama, específicamente en la porosidad de la matriz, presentando un incremento de sus cavidades, y con ello, se materializa la disminución en la unión de las partículas del producto frito (Yang et al., 2017). Con los tiempos de procesamiento utilizados no se encontraron diferencias significativas entre los valores medios de cohesividad, indicando igualdad en la fuerza de unión entre las partículas de la ahuyama frita. De forma general, los resultados obtenidos apuntan a que la ahuyama tiende a recuperar su estructura un $38 \%$ como máximo referente a su estado inicial posterior a la primera compresión realizada, significando que el proceso de freído logra disminuir la fuerza con las que las partículas estructurales de la ahuyama están unidas (Guzmán et al., 2015).

Finalmente, para la variable masticabilidad se obtuvo que con las temperaturas de proceso empleadas no se marcaron diferencias estadísticas entre los valores del requerimiento energético necesario para masticar el producto a base de ahuyama y desintegrarlo hasta que pueda ser ingerido por el consumidor; con respecto a los niveles de tiempo de proceso se puede inferir que a medida que el tiempo de proceso transcurrió los valores de masticabilidad fueron disminuyendo hasta marcar diferencias significativas, el valor que permitió obtener un menor valor más bajo de esta variable fue el tiempo de proceso de $160 \mathrm{~s}$, permitiendo obtener un producto que requerirá menor energía por parte de la acción de los molares del consumidor para triturar la ahuyama frita y desintegrarla hasta que se pueda ingerir totalmente (González et al., 2015).

\section{CONCLUSIONES}

De los resultados, análisis y discusiones realizadas, es posible inferir que el fenómeno de transferencia de masa que se efectúa durante el proceso de freído de la ahuyama, produce modificaciones en algunas de las características evaluadas en el análisis de perfil de textura. Por ende, el incremento de la temperatura y el tiempo de proceso disminuyen la dureza permitiendo obtener productos menos duros por efecto de la gelatinización de almidones cuando entran en contacto con un aceite más caliente y por mayor tiempo de exposición al intercambio másico. Referente a la adhesividad, el incremento de estos factores representa obtener productos mucho menos pegajosos que requerirán menor trabajo por parte del consumidor para lograr despegar la ahuyama frita de una superficie, ya sea de los molares o el paladar. En la cohesividad, el incremento de la temperatura permite obtener productos con mayor resistencia a la fragmentación por efecto de la acción mecánica del equipo de textura. En la elasticidad, las temperaturas y tiempos evaluados fragmentaron igual estructura de la ahuyama frita. Finalmente, para la masticabilidad las temperaturas de proceso empleadas permiten obtener productos que requerirán igual energía para ser masticados y desintegrados hasta que pueda ser ingerido por el consumidor, de igual forma, con el tiempo de proceso de 160 s se logra el menor valor de esta característica. De forma general, el proceso de freído por inmersión puede considerarse como una alternativa de procesamiento de la ahuyama, puesto que genera cambios deseables en los atributos de textura, principalmente en la dureza, adhesividad, cohesividad y en la masticabilidad. 


\section{REFERENCIAS}

Adedeji, A. y M. Ngadi, Impact of freezing method, frying and storage on fat absorption kinetics and structural changes of parfried potato, Journal of Food Engineering, 218, 24-32 (2018)

Alvis, A., P. Romero y otros tres autores, Evaluación del color, las propiedades texturales y sensoriales de salchicha elaborada con carne de babilla (Caiman Crocodilus Fuscus), Revista chilena de nutrición, 44(1), 89-94 (2017)

Alvis-Bermúdez, A., P. Romero-Barragán y G. Arrazola-Paternina, Pérdida de humedad y absorción de aceite durante fritura de tajadas de plátano (musa paradisiaca I.), Biotecnología en el Sector Agropecuario y Agroindustrial (2016)

AOAC, Official Methods of Analysis, 312-313, Washington DC, USA (2003)

Arias-Méndez, A., A. Warning, A.K. Datta y E. Balsa-Canto, Quality and Safety Driven Optimal Operation of Deep-Fat Frying of Potato Chips, doi:10.1016/j.jfoodeng.2013.05.001, Journal of Food Engineering, 119(1),125-134 (2013)

Arturo-Suarez, E., P. Paz-Peña y otros tres autores, Efecto del sistema de producción en la maduración fisiológica de cucúrbita Moschata var. Bolo verde, Biotecnología en el Sector Agropecuario y Agroindustrial, 14(2), $29-37$ (2016)

Barati, F. y H. Talebi, A new efficient MEP.1 series for $2 f$ factorial experiments, Journal of Statistical Planning and Inference (2018)

Barrios-Barrios, L., O. Osorio-Mora y A.F. Cerón-Cárdenas, Estudio de las cinéticas de pérdida de agua y absorción de aceite durante la fritura de arveja (Pisum sativum L.), Acta Agronómica, 65(3), 226-231 (2016)

Brannan, R.G., E. Mah y otros cinco autores, Influence of ingredients that reduce oil absorption during immersion frying of battered and breaded foods, European journal of lipid science and technology, 116(3), 240-254 (2014)

Duque, M., Prácticas en el Consumo de Frutas y Hortalizas en Hogares de dos Comunidades de la Zona Rural del Municipio de Turbo, 1-45 (2013)

González, A., A. Alvis y G. Arrázola, Efecto del Recubrimiento Comestible en las Propiedades de Trozos de Batata (Ipomoea Batatas Lam) Fritos por Inmersión: Parte 1: Textura, doi:10.4067/S0718-07642015000100011, Información Tecnológica, 26(1), 95-102 (2015)

Granados, C., D. Acevedo, A. Cabeza y A. Lozano, Análisis de Perfil de Textura en Plátanos Pelipita, Hartón y Topocho, doi: 10.4067/S0718-07642014000500006, Información Tecnológica, 25(5), 35-40 (2014)

Guzmán, L., D. Acevedo y D. Tirado, Deshidratación osmótica de pulpa de tamarindo (Tamarindus indica I.): influencia de la temperatura y la concentración, Revista Udca Actualidad \& Divulgación Científica, 17(1), 123-130 (2015)

Jacobo-Valenzuela, N., J. Zazueta-Morales y otros cuatro autores, Rediscovering winter squash (Cucurbita moschata d.) cv. cehualca as a magic food in sinaloa state, doi:10.1016/j.foodres.2011.04.039, Revista Salud Pública y Nutrición, 1, 214-219 (2008)

Jacobo-Valenzuela, N., J. Zazueta-Morales y otros cinco autores, Chemical and physicochemical characterization of winter squash (Cucurbita moschata D.), Notulae Botanicae Horti Agrobotanici Cluj-Napoca, 39(1), 34 (2011)

Kan, L., Q. Li y otros cuatro autores, Effect of thermal processing on the physicochemical properties of chestnut starch and textural profile of chestnut kernel, doi:10.1016/j.carbpol.2016.06.008, Carbohydrate polymers, 151, 614623 (2016)

Lee, C.H., J.K. Cho y otros cuatro autores, Enhancing $\beta$-carotene Content in Asian Noodles by Adding Pumpkin Powder, doi: 10.1094/CCHEM.2002.79.4.593, Cereal Chemistry, 79 (4), 593-595 (2002)

Márquez, M., C. Yépez, R. Sútil- Naranjo y M. Rincón, Aspectos básicos y determinación de las vitaminas antioxidantes E y A. Investigación clínica, 43(3), 12-22 (2002)

Mba, O. I., M.J. Dumont y M. Ngadi, Thermostability and degradation kinetics of tocochromanols and carotenoids in palm oil, canola oil and their blends during deep-fat frying, LWT-Food Science and Technology, 82, 131-138 (2017)

Montes, N., I. Millar y otros cinco autores, Absorción de aceite en alimentos fritos, Revista chilena de nutrición, 43(1), 87$91(2016)$

Moreno, M. C. y P. Bouchon, A different perspective to study the effect of freeze, air, and osmotic drying on oil absorption during potato frying, Journal of Food Science, 73(3) (2008)

Oloyede, F. M., G.O. Agbaje, E.M. Obuotor e I.O. Obisesan, Nutritional and antioxidant profiles of pumpkin (Cucurbita pepo Linn.) immature and mature fruits as influenced by NPK fertilizer, Food chemistry, 135(2), 460-463 (2012)

Oyedeji, A. B., O.P. Sobukola y otros cinco autores, Effect of frying treatments on texture and colour parameters of deep fat fried yellow fleshed cassava chips, Journal of Food Quality (2017)

Pardo, A., J. Garrido, M.A. Ruiz y R. San Martín, La interacción entre factores en el análisis de varianza: errores de interpretación, Psicothema, 19(2) (2007)

Pedreschi, F. y P. Moyano, Oil uptake and texture development in fried potato slices, Journal of Food Engineering, 70(4), 557-563 (2005) 
Quintana, S.E., R.M. Marsiglia y otros tres autores, Chemical Composition and Physicochemical Properties of Squash (Cucurbita moschata) Cultivated in Bolivar Department (Colombia) (2018)

Rodríguez-Manrique, J., A. Alvis-Bermúdez y otros cuatro autores, Pérdidas de humedad y absorción de aceite en ahuyama sometida a osmodeshidratación y fritura, Agronomía Colombiana, 34(1Supl), S332-S335 (2016)

See, E., W. Abdullah, W. Nadiah y A. Abdul, Physico-chemical and sensory evaluation of breads supplemented with pumpkin flour, Asean Food Journal 14(2), 123-130 (2007)

Yang, J., A. Martin, S. Richardson y C.H. Wu, Microstructure investigation and its effects on moisture sorption in fried potato chips, Journal of Food Engineering, 214, 117-128 (2017)

Zhang, Q. C. Wan y otros siete autores, Evaluation of the non-aldehyde volatile compounds formed during deep-fat frying process, Food Chemistry, 243, 151-161 (2018) 
